\title{
Modelo factorial en la motivación laboral y desempeño docente. Caso: Institución Educativa Emblemática Isabel la Católica, Lima - Perú
}

\section{Factor model in work motivation and teacher performance. Case: Institución Educativa Emblemática Isabel la Católica, Lima - Perú}

Luis Alberto Geraldo Campos, ${ }^{\text {a }}$ Juan Jesus Soria Quijaite, ${ }^{2}$ Margorie Joyce Rosello Jauregui ${ }^{3}$ y Katherine Margoth Buendia Herrera ${ }^{4}$

EP Administración, Universidad Peruana Unión, Lima, Perú ${ }^{134}$

EP Ingeniería de Sistemas, Universidad Peruana Unión, Lima, Perú

iD Orcid ID: https://orcid.org/0000-0002-8366-689X ${ }^{1}$

(iD) Orcid ID: https://orcid.org/0000-0002-4415-8622

(iD) Orcid ID: https://orcid.org/0000-0002-4821-7095 3

(iD) Orcid ID: https://orcid.org/0000-0001-7801-2341

Recibido: 20 de julio de 2020

Aceptado: 25 de octubre de 2020

\section{Resumen}

Se ha diseñado esta investigación con el objetivo de analizar los indicadores de la motivación y desempeño docente para determinar un modelo factorial de predicción en docentes. La metodología corresponde a un enfoque cuantitativo de carácter predictivo, para esto se utilizó dos instrumentos y se aplicaron a dos poblaciones: el instrumento motivación fue aplicado a los docentes y el instrumento de desempeño docente, a los estudiantes del 4to y 5to año del nivel secundario. Los participantes estuvieron conformados por 59 docentes y 197 estudiantes. El índice KMO fue de 0.636, esto permitió realizar un análisis factorial que sigue un proceso metodológico de siete fases bien definidas. Los resultados arrojaron tres clústeres: la motivación extrínseca y trascendental (clúster 1); motivación intrínseca (clúster 2); y desempeño docente (clúster 3) en la que se obtuvo dos eigenvalores mayores que 1 permitiendo proponer dos modelos de componentes principales que predicen los indicadores de la variable de estudio.

Palabras clave: Análisis factorial, motivación, desempeño docente, ACP, modelo factorial. 


\begin{abstract}
This research has been designed with the objective of analyzing the indicators of teacher motivation and performance to determine a factorial model of prediction in teachers. The methodology corresponds to a quantitative approach of a predictive nature, for this, two instruments were used and they were applied to two populations: the motivation instrument was applied to teachers and the teacher performance instrument, to students of the 4th and 5th year of secondary level. The participants were made up of 59 teachers and 197 students. The KMO index was 0.636, this allowed a factor analysis that follows a methodological process of seven well-defined phases. The results yielded three clusters: extrinsic and transcendental motivation (cluster 1); intrinsic motivation (cluster 2); and teaching performance (cluster 3) in which two eigenvalues greater than 1 were obtained, allowing us to propose two main component models that predict the indicators of the study variable.
\end{abstract}

Keywords: Factorial analysis, motivation, teaching performance, ACP, factorial model.

\title{
Introducción
}

El docente es un profesional comprometido, sus acciones y desempeño se fundamentan en los procesos pedagógicos científicos, como cualquier otro colaborador, necesita estar motivado, deben ser evaluados por sus desempeños y monitoreados en el desarrollo de sus actividades académicas. Los directivos o el jefe inmediato de las Instituciones Educativas (IE) son los encargados de evaluar el desempeño y la motivación de los docentes, con el propósito de brindar a los estudiantes y a la institución en general, resultados óptimos en el desempeño académico.

La motivación es un proceso complejo que canaliza energías y tiene un impacto en como las personas persiguen sus objetivos e intereses y la forma para lograrlos (López-Arellano, VélezSalazar, \& Franco-López, 2017). En el campo pedagógico, la motivación es una característica que adquiere el maestro para mantener activamente una práctica educativa de calidad, esto transmite al estudiante para obtener logros basados en su formación cognitiva, actitudinal y psicológica (RojasSegovia \& Romero-Varela, 2019). Esto significa que, la motivación docente, es fundamental para conseguir la anhelada satisfacción laboral y afirmar el desarrollo de los procesos pedagógicos, a tal punto de superar la satisfacción de determinadas necesidades básicas del docente (Franco, López, \& Vélez, 2015).

La conducta y el desempeño de las personas lo determina su nivel de motivación, lo que permitirá mejoras en su nivel de eficacia en las funciones a desempeñar (López-Arellano et al., 2017). La motivación del docente recae en el placer por enseñar y compartir conocimientos con los aprendices, esta se traduce como motivación intrínseca, además de los motivadores extrínsecos 
relacionados a los premios y satisfacciones que puede recibir de los estudiantes (Rodríguez-Pérez, 2012).

En ese sentido, las motivaciones han sido agrupadas en dos componentes: en primer lugar se encuentran las motivaciones internas que parten de necesidades, las cogniciones y emociones de las personas; en cambio las motivaciones externas pretenden determinar el comportamiento de los estímulos o el conjunto de hechos externos que permiten cambiar la conducta a partir de consecuencias sociales, ambientales y culturales (López-Arellano et al., 2017). Sin embargo, existe otra componente que ayuda a explicar mejor la motivación, por lo que se añade la motivación trascendente, la cual hace referencia a la consecuencia de una determinada acción que un individuo realiza y tiene un efecto en los demás indirectamente (Cedeno, 2004; Sánchez, 2019; Sanizaca, 2018; Schnitker, Gilbertson, Houltberg, Hardy, \& Fernandez, 2020).

Estudios realizados demuestran que los principales factores de motivación enfocados a los docentes están los alineados con la motivación intrínseca, en específico la vocación (López-Arellano et al., 2017). La motivación intrínseca es la que inspira a hacer cosas por el simple gusto de hacerlas, surge del interior de la persona con el fin de satisfacer deseos no materiales y sin obtener ningún incentivo del exterior, por lo que se espera que las personas estén siempre motivadas extrínsecamente con el objeto de realizar actividades con responsabilidad, autonomía y competencia (Jiménez \& Bueno, 2003).

Por su parte, Chiavenato (2005) se refiere a los factores intrínsecos de la teoría de Herzberg que estos se relacionan con el cargo y con la naturaleza de las tareas que un colaborador realiza. Estos factores motivacionales se encuentran bajo control de la persona, está relacionado con aquello que el individuo hace y desempeña. Si bien la motivación intrínseca es un componente importante para el desarrollo del talento humano, la componente motivación extrínseca también lo es, fundamentalmente en los niveles de educación secundaria, por el hecho de presentar desempeños sobresalientes, importante para insertarse al mundo universitario (Blumen, 2008).

"La motivación trascendente es aquella que se genera cuando la persona encuentra su satisfacción profesional en el servicio y en la ayuda a sus colaboradores" (Ugarte \& Naval, 2010, p. 8). Además, la motivación trascendente ayuda hacia otras personas en su conjunto indirectamente y tiene conexiones más sutiles con principios éticos y sociales (Silva, 2010; Sucuoglu, 2017). En el sentido educativo la motivación transcendente está relacionada al cumplimiento de metas institucionales, la vocación del servicio e integración a la comunidad (Sánchez, 2019). 
La motivación trascendente promueve el bienestar del personal que se refleja en los desempeños efectivos. Además, está más relacionado al aspecto cognitivo, bajo una cosmovisión de creencias, valores y principios del individuo, lo que lleva a un bienestar total y al deseo de aprender (Guerrero, La Rosa, \& Díaz, 2015; Schnitker et al., 2020).

El desempeño docente se concibe como la práctica pedagógica visible, manifestándose cuando el docente expresa su competencia y aprendizajes esperados (Gálvez \& Milla, 2018). Además lo constituyen las acciones que lleva a cabo en el aula e incluyen las acciones de planificación y elección de material; asimismo, el conjunto de creencias actitudes y expectativas dentro de su entorno de desempeño pedagógico (Sánchez-Claros, 2014).

El docente dentro de su desempeño tiene como finalidad ser retroalimentado y capacitado para mejorar la calidad de enseñanza (Gómez \& Valdés, 2019). Los docentes consideran que existen cuatro actores principales que determinan la calidad educativa, los cuales hacen referencia a la escuela, contexto, docente y gobierno, estos deben trabajar de forma colaborativa para lograrla (Martínez-Chairez, Guevara-Araiza, \& Valles-Ornelas, 2016).

El docente tiene cuatro competencias necesarias para desempeñarse adecuadamente, competencias académicas, administrativas y sociales, estas permiten actuar con eficiencia en el desempeño de cada una de las funciones encomendadas (Pacheco, 2016). Para Martínez \& Carmona (2009) las competencias son una suma de comportamientos que algunos individuos dominan mejor que otros, que les permite destacar en una situación determinada.

La competencia no se limita a los elementos cognitivos basados en conocimientos, sino que también involucra aspectos funcionales que determinan habilidades técnicas especializadas, así como atributos interpersonales y los valores éticos que le permiten desenvolverse en contextos específicos (Centro Europeo para el Desarrollo de la Formación Profesional, 2010; Oficina Internacional del Trabajo, 2005). Para Echeverría (2002) en su estudio titulado gestión de la competencia de acción profesional, plantea desde una visión holística cuatro competencias que van desde competencia técnica, metodológica, participativa y personal, el cual, están basadas en el saber, saber hacer, saber estar y saber ser respectivamente.

La competencia técnica, es aquella que posee conocimientos especializados de acuerdo a su ámbito profesional y tiene la destreza del dominio de los contenidos y tareas relacionadas con su actividad profesional. La metodológica se basa en el saber hacer, es decir saber aplicar los conocimientos a situaciones reales con procedimientos correctos, así como solucionar problemas y 
plasmar con ingenio a nuevas situaciones. La participativa se enfoca al saber estar, es decir, estar actualizado en el constante cambio laboral, predispuesto y dispuesto al entendimiento interpersonal, a la comunicación, cooperación y demostrando un comportamiento participativo con los demás. Finalmente, la competencia técnica se enfoca en el saber ser, es decir, tener una percepción bien definida de sí mismos, actuando de acuerdo a las convicciones de manera responsable y sobrellevando las frustraciones (Aneas, 2003; Echeverría, 2002).

Indudablemente existen diversos estudios que abordan los constructos de motivación y desempeño. Sin embargo, existen escasas investigaciones que indiquen que una buena motivación ayude a predecir buenos resultados en el desempeño docente. Viendo esta necesidad, el presente estudio trata de llenar este vacío, estudiando la motivación y desempeño docente, este último desde las competencias propuestas según la teoría de Echeverría (2002): competencia técnica, metodológica, participativa y personal.

La investigación se centra en un estudio cuantitativo de carácter predictivo a través de un análisis factorial, con el propósito de analizar los indicadores de la motivación y desempeño docente. El análisis permitirá agrupar mediante clúster las dimensiones de motivación centrados en motivación intrínseca, extrínseca y trascendental con el constructo desempeño docente, con la finalidad de determinar un modelo factorial que ayude a predecir su comportamiento.

\section{Metodología}

La investigación corresponde a un enfoque cuantitativo de carácter predictivo, en la cual participaron 59 docentes y 197 estudiantes del 4to y 5to año del nivel secundario de la Institución Educativa Emblemática Isabel la Católica, La Victoria, Lima, Perú. En ella se aplicaron dos instrumentos que estuvieron organizados por la motivación y el desempeño docente, ambos instrumentos fueron construidos por Velasco, Bautista, Sánchez, \& Cruz (2012), validados y adaptados por juicio de expertos al contexto peruano (Bruno, 2017). El primer instrumento de motivación se aplicó a los docentes, este agrupa tres dimensiones: motivación intrínseca (6 ítems), extrínseca (6 ítems) y trascendental (6 ítems), la cual están valoradas en una escala de Likert del 1 al 5, siendo 1 igual a nunca y 5 igual a siempre, con una confiabilidad de Alfa de Cronbach de 0.709 en el estudio de Bruno (2017) y para el presente estudio con un Alfa de 0.810 (ver tabla 1). El segundo instrumento para el desempeño docente se aplicó a estudiantes del 4to y 5to año. Cabe señalar que los autores para la elaboración del instrumento partieron de la teoría de Echeverría (2002), creando 23 reactivos y agrupándolas en 4 dimensiones: competencias técnicas (8 ítems), 
competencia metodológica (6 ítems), competencia personal (5 ítems) y la competencia participativa (4 ítems), con una valoración de escala Likert del 1 al 5, donde 1 es igual a muy en desacuerdo y 5 totalmente de acuerdo. El resultado de análisis de fiabilidad de Alfa Cronbach del instrumento arrojó un valor de $\alpha=0.936$ como se muestra en la tabla 1 .

\section{Tabla 1}

Estadísticas de fiabilidad de las variables de estudio

\begin{tabular}{|c|c|c|}
\hline Indicador & Alfa de Cronbach & $\mathrm{N}$ de elementos \\
\hline Motivación docente & .810 & 18 \\
\hline Desempeño docente & .936 & 23 \\
\hline
\end{tabular}

Previo a la aplicación de los instrumentos se presentó una solicitud de autorización a la dirección de la IEE. Isabel la Católica cuya respuesta fue positiva tardando solo una semana en dar respuesta al documento presentado, dando la autorización y facilidades para ingresar a la institución y aulas de clase con la finalidad de proceder a la aplicación de los instrumentos.

Cabe precisar que la recolección de datos fue desde dos perspectivas: el instrumento motivación se aplicó a los docentes para conocer el nivel de motivación, asimismo el instrumento desempeño docente se aplicó para conocer desde la visión del estudiante el nivel del desempeño docente. Para un análisis más profundo y cumplir con los propósitos del presente estudio, se procedió a realizar un análisis siguiendo las fases del proceso metodológico (ver figura 1).

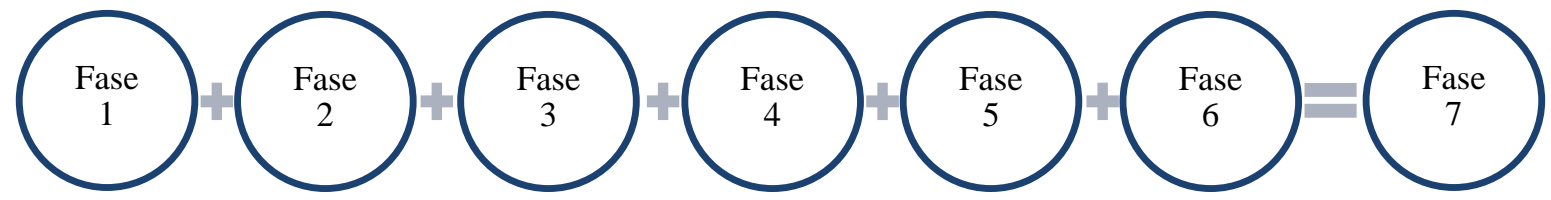

Figura 1. Fases del proceso metodológico empleado

\section{Fase 1. Recolección y limpieza de datos}

Luego de la aplicación del instrumento a los estudiantes y docentes de la institución educativa se prepararon tres archivos en Excel con el propósito de limpiar los datos y pasarlos al software SPSS vs25 y Minitab vs18. Luego, se analizaron las dimensiones e ítems de los instrumentos determinando el mayor y menor puntaje de forma descriptiva, filtrando los valores atípicos de la información. Además, se procedió a la baremación por dimensiones de los ítems y posteriormente se determinaron 
los niveles de las variables de estudio.

\section{Fase 2. Transformación y normalización de datos}

Luego se procedió a la transformación de los datos agrupándolos por dimensiones para realizar un análisis de normalización de datos, obteniendo los resultados de K-S mostrados en la tabla 2.

\section{Fase 3. Selección y preparación de datos}

Una vez los datos limpiados, transformados y normalizados, se procedió a obtener los valores propios, el cual se tomaron aquellos que son mayores que 1, obteniéndose una reducción de dimensión en el plano Cartesiano. Asimismo, se tuvo en cuenta la varianza total explicada en la toma de decisión en función a los valores propios.

\section{Fase 4. Cálculo de matriz de covarianza}

Se calculó el índice KMO, con la finalidad de comparar las magnitudes de los coeficientes de correlación parcial, que cuanto más pequeño es su valor, mayor será el coeficiente de correlación parcial, en el caso de nuestro estudio asciende a 0.64, esto es aceptable (Catena, Ramos, \& Trujillo, 2003; César, 2004). Además, se determinó la matriz de covarianza para la relación entre los ítems y las dimensiones de las variables, seleccionándose los coeficientes de correlación mayores a 70\% de la información de la varianza explicada (César, 2004).

\section{Fase 5. Calcular valores y vectores propios}

Se determinaron los valores y vectores propios con las características mayores a 1 y por conveniencia se eligieron dos de ellos, para su interpretación en el plano Cartesiano.

\section{Fase 6. Ordenamiento de los valores propios}

Previo a la corrida de los datos a través del software, se solicitó de forma creciente el ordenamiento de los valores propios, con la finalidad de tomar la mejor componente para la presente investigación. 


\section{Fase 7. Representación de nuevos conjuntos de datos}

Una vez rotado con la técnica Varimax el conjunto de datos, se obtuvieron las dos componentes principales, reduciendo la dimensión en el plano cartesiano mostrado en la figura 8.

\section{Resultados}

\section{Análisis del nivel de motivación docente}

La figura 2 muestra los resultados sobre el nivel de motivación, el 35.60\% muestran un nivel bajo, el $32.20 \%$ un nivel medio y alta de motivación en los docentes.

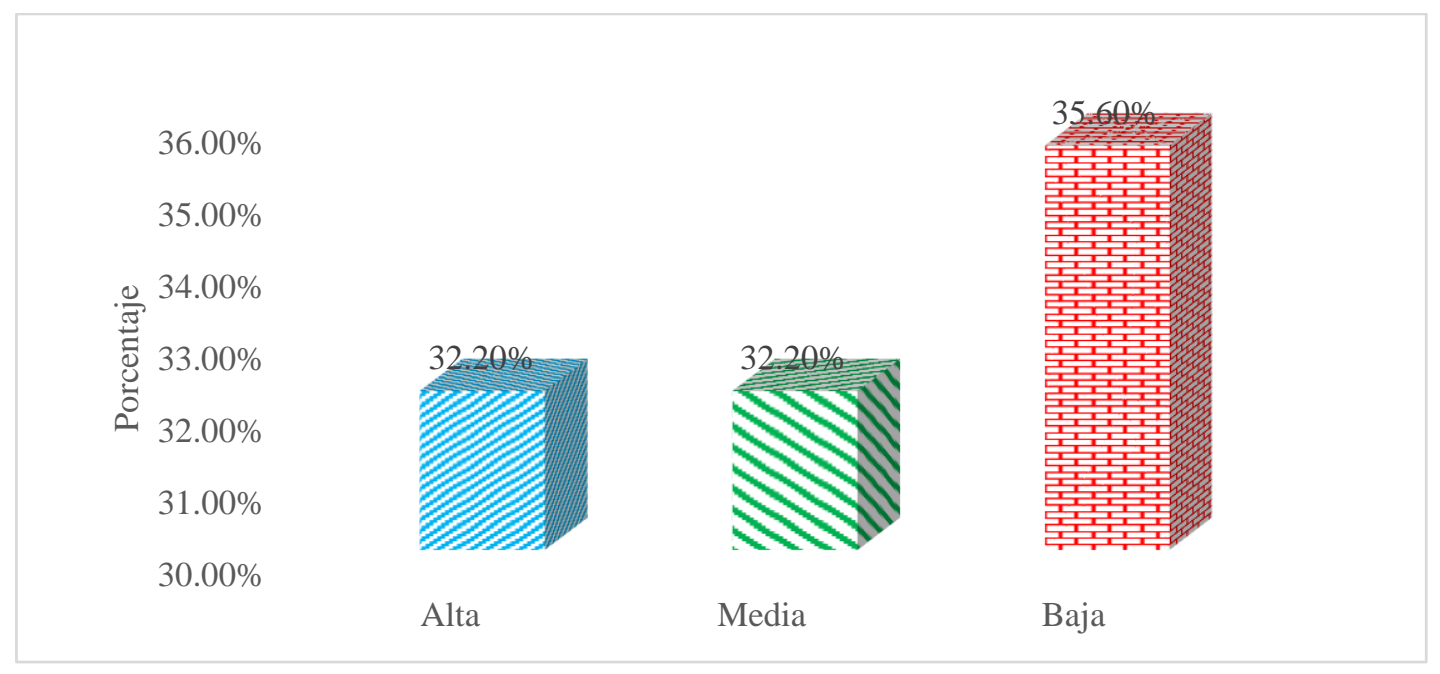

Figura 2. Nivel de motivación docente

\section{Análisis del nivel de desempeño docente}

Los resultados de la figura 3 revelan que el 34.52\% tienen un nivel de desempeño alto, seguido del $34.01 \%$ de nivel de desempeño medio y 31.47 con un nivel de desempeño bajo. 


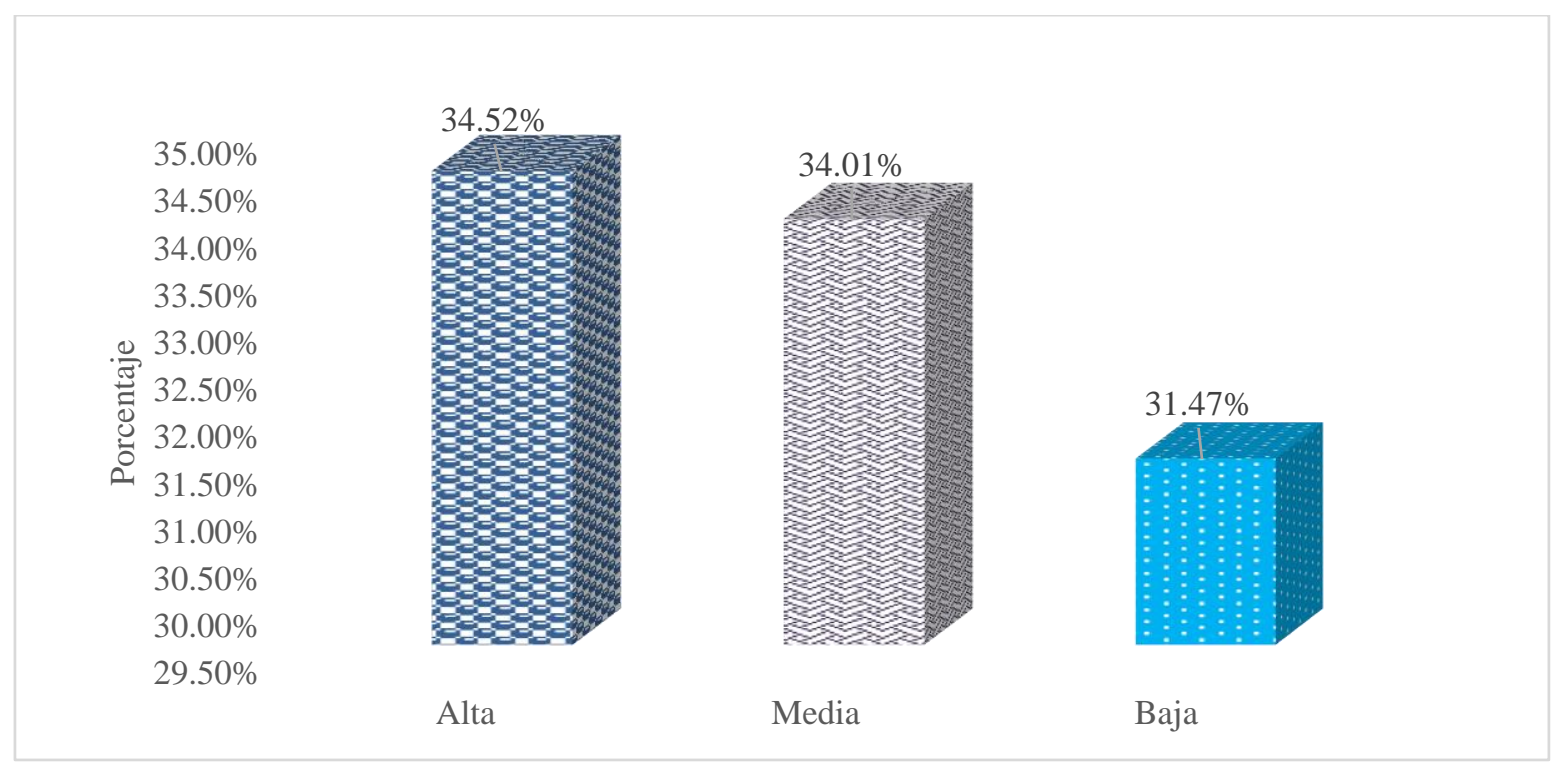

Figura 3. Nivel de desempeño docente

\section{Análisis de normalidad de variables}

$\mathrm{H}_{0}$ : La variable no está normalmente distribuida

$\mathrm{H}_{1}$ : La variable esta normalmente distribuida

Se rechaza la $\mathrm{H}_{\mathrm{o}}$ si el sig. bilateral es menor de 0.05 (nivel de significancia)

\section{Tabla 2}

Pruebas de normalidad $K-S$

\begin{tabular}{lccc}
\multicolumn{2}{c}{ Kolmogorov-Smirnov $^{\mathrm{a}}$} & & \\
\hline & Estadístico & $\mathrm{gl}$ & Sig. \\
Motivación docente &, 093 & 59 &, $200^{*}$ \\
Desempeño docente &, 162 & 59 &, 001 \\
*. Esto es un límite inferior de la significación verdadera. & & \\
a. Corrección de significación de Lilliefors & &
\end{tabular}

Analizando las puntuaciones de las variables del estudio se determinó que el sig. bilateral para la motivación docente es $0.200^{*}$, que por ser mayor $\alpha=0.05$ de significancia la variable si tiene normalidad y para el desempeño docente su sig. bilateral es de 0.001 , que por ser menor al nivel de significancia alfa no tiene normalidad. Se concluye que al no comportarse las dos variables de forma normal, se optó realizar la prueba estadística no paramétrica de U de Mann-Whitney, por ser una de 
las pruebas no paramétricas más potentes disponibles (Lewis-Beck, Bryman, \& Futing, 2004)

\section{Prueba de hipótesis $U$ de Mann-Whitney}

Ho: NO existe diferencia significativa entre la motivación y el desempeño de los docentes

$\mathrm{H}_{1}$ : Existe diferencia significativa entre la motivación y el desempeño de los docentes

\section{Hipótesis estadísticas}

$$
\left\{\begin{array}{l}
H_{0} \cdot M e_{M D}=M e_{D D} \\
H_{1} \cdot M e_{M D} \neq M e_{D D}
\end{array}\right.
$$

Donde:

$\mathrm{MD}=$ Motivación docente

$\mathrm{DD}=$ Desempeño docente

$\mathrm{Me}=$ Mediana de los datos

\begin{tabular}{|llll|}
\hline \multicolumn{1}{|c}{ Hipótesis nula } & Prueba & Sig. & Decisión \\
\hline 1 & $\begin{array}{l}\text { Las medianas de Puntuaciones son } \\
\text { las mismas entre las categorías de } \\
\text { Factores. }\end{array}$ & $\begin{array}{l}\text { Prueba de la } \\
\text { mediana para } \\
\text { muestras } \\
\text { independientes }\end{array}$ &, $000 \begin{array}{l}\text { Rechazar la } \\
\text { hipótesis } \\
\text { nula. }\end{array}$ \\
\hline 2 & $\begin{array}{l}\text { Prueba U de } \\
\text { Mann-Whitney } \\
\text { para muestras } \\
\text { la misma entre las categorías de } \\
\text { Factores. }\end{array}$ &, $000 \begin{array}{l}\text { Rechazar la } \\
\text { hipótesis } \\
\text { nula. }\end{array}$ \\
\hline
\end{tabular}

Se muestran significaciones asintóticas. El nivel de significación es de ,05.

Figura 4. Prueba de hipótesis de la U de Mann-Whitney

En la figura 4, se observa el sig. bilateral de la variable motivación y desempeño docente, está es de 0.000 que por ser menor al nivel de significancia $\alpha=0.05$ rechazamos la hipótesis nula $\mathrm{H}_{0}$ y aceptamos la hipótesis alterna $\mathrm{H}_{1}$, lo que confirma que existe diferencia significativa entre la motivación y desempeño de los docentes, con una gran mediana igual a 94 para ambos grupos (figura 5), en la que la mayor relevancia está en el grupo 2 con un $24.24 \%$ de eficiencia en el desempeño docente (ver figura 6). 


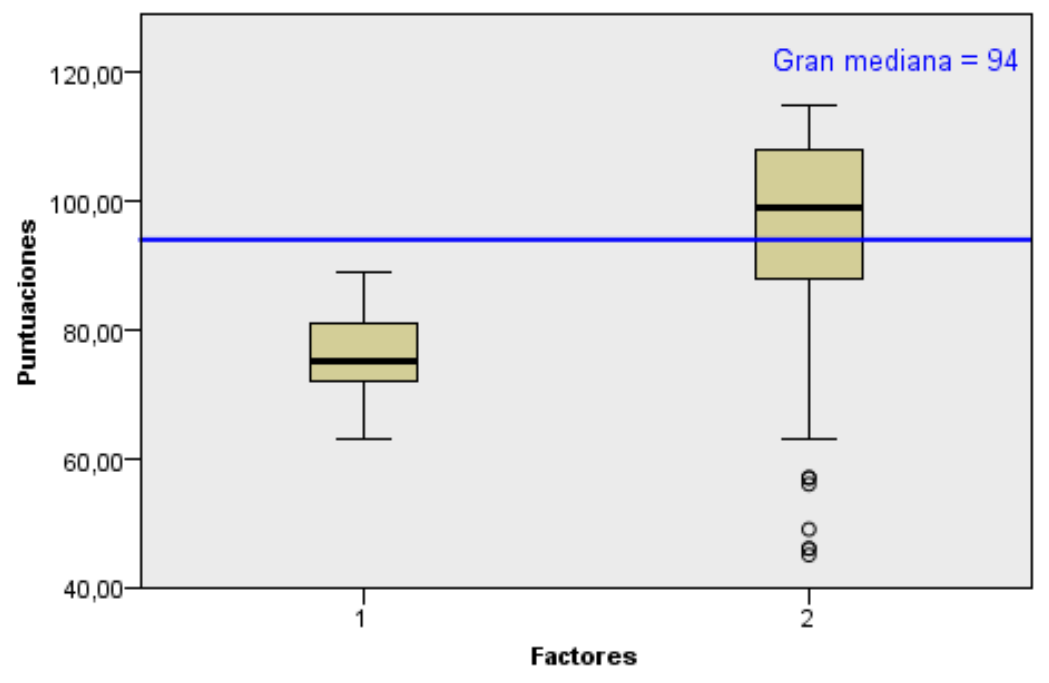

Figura 5. Diagrama de caja de motivación y desempeño docente

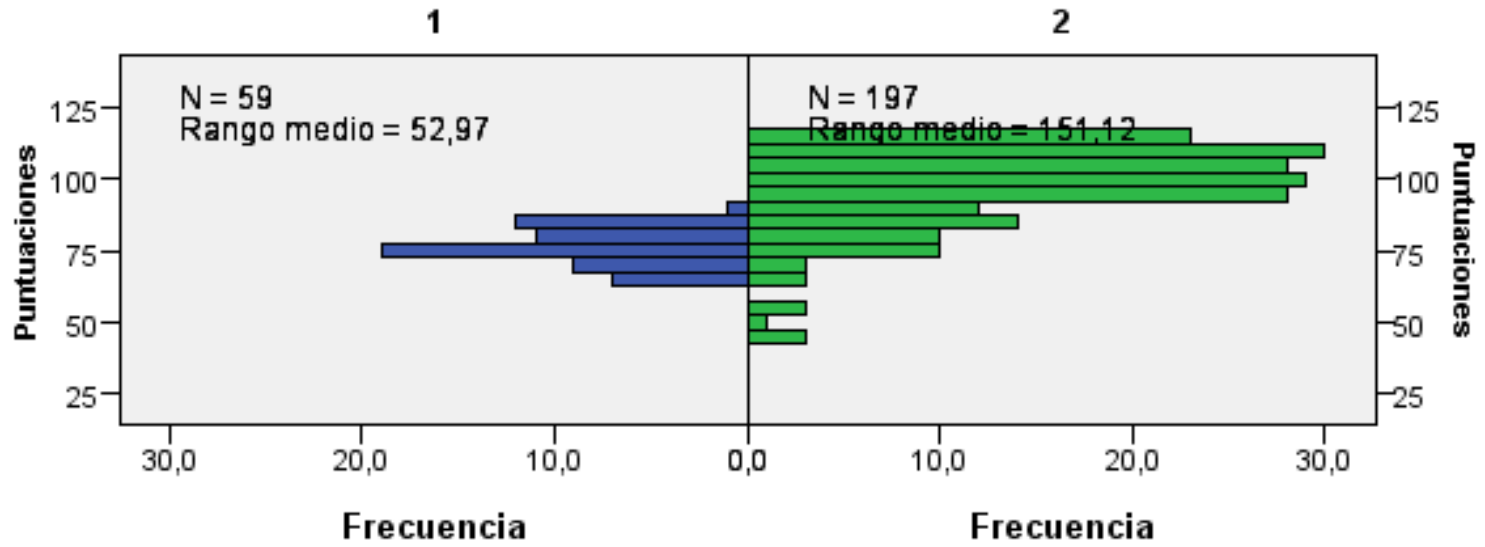

Figura 6. Puntuaciones de los factores de docentes (1) y alumnos (2)

$$
\begin{gathered}
Z=\frac{U-0.5\left(n_{x} n_{y}\right)}{\sqrt{\frac{n_{x} n_{y}\left(n_{x}+n_{y}+1\right)}{12}}} \\
Z=\frac{10267.500-0.5(59 \times 197)}{\sqrt{\frac{59 \times 197(59+197+1)}{12}}}=8.93
\end{gathered}
$$

Los resultados de la figura 7, más el cálculo de la fórmula 2 dio un valor de $\mathrm{Z}$ calculado igual 
a 8.93, este es mayor al valor de Z crítico igual a 1.96, entonces se rechaza la hipótesis nula $\mathrm{H}_{0}$ y se acepta la hipótesis alterna $\mathrm{H}_{1}$, es decir, la motivación laboral influye significativamente en el desempeño docente a un nivel de significancia $\alpha=0.05$ y un nivel de confiabilidad del 0.95 .

\begin{tabular}{|lr|}
\hline N total & 256 \\
\hline U de Mann-Whitney & $10.267,500$ \\
\hline W de Wilcoxon & $29.770,500$ \\
\hline Estadístico de contraste & $10.267,500$ \\
\hline Error estándar & 498,766 \\
\hline $\begin{array}{l}\text { Estadístico de contraste } \\
\text { estandarizado }\end{array}$ & 8,934 \\
\hline Sig. asintótica (prueba bilateral) &, 000 \\
\hline
\end{tabular}

Figura 7. Resumen de la prueba de U de Mann-Whitney

\section{Análisis Factorial}

Análisis de componentes principales

\section{Tabla 3}

Prueba de KMO y Bartlett

Prueba de KMO y Bartlett

\begin{tabular}{lcc}
\hline Medida Kaiser-Meyer-Olkin de adecuación de muestreo &, 636 \\
& Aprox. Chi-cuadrado & 1726,639 \\
& Gl & 820 \\
Prueba de esfericidad de Bartlett & Sig. &, 000
\end{tabular}

En la tabla 3 se observa que la prueba de Medida Kaiser-Meyer-Olkin tiene un valor de 0.636 de adecuación de muestreo, de acuerdo a (Catena et al., 2003; César, 2004) hace que el estudio sea adecuado para el análisis factorial de las variables en estudio. Además, se observa un sig. bilateral de 0.000 que confirma la significancia de la homocedasticidad de las variables. 


\section{Tabla 4}

Varianza total explicada de los componentes

\begin{tabular}{ccccccc} 
Componente & \multicolumn{3}{c}{ Sumas de cargas al cuadrado de la } \\
extracción & \multicolumn{3}{c}{ Sumas de cargas al cuadrado de la rotación } \\
\hline & Total & $\%$ de & $\%$ & Total & $\%$ de & $\%$ \\
1 & varianza & acumulado & & varianza & acumulado \\
2 & 12,368 & 30,166 & 30,166 & 12,368 & 30,166 & 30,166 \\
& 3,872 & 9,443 & 39,609 & 3,872 & 9,443 & 39,609
\end{tabular}

Método de extracción: análisis de componentes principales.

En la tabla 4, se observa que el $39.61 \%$ de la varianza total explicada, representada por dos valores propios mayores que 1 y con la técnica de rotación Varimax se redujo a dos componentes.

\section{Modelos factoriales de la investigación}

El análisis factorial es una clase de procedimientos utilizados para la reducción y el resumen de datos. Es un proceso de interdependencia en el que se examina un conjunto de interacciones interdependientes. Cada variable se expresa como una combinación lineal de factores subyacentes (Saini, Kumar, \& Kaur, 2020). El modelo de factores se representa en la ecuación 3:

$F_{i}=A_{i 1} X_{1}+A_{i 2} X_{2}+A_{i 3} X_{3}+\ldots .+A_{i m} X_{m}$

Donde:

$F_{i}=$ Estimador de cada factor

$\mathrm{X}_{\mathrm{i}}=$ Puntuaciones de los coeficientes de los factores

$\mathrm{m}=$ número de variables

\section{Clúster de los indicadores}

En la figura 8, se observa que con la técnica de análisis de componentes principales se han clasificado en tres grupos respecto a la motivación y desempeño, tal como sigue: motivación extrínseca y trascendental (clúster 1), motivación intrínseca (clúster 2), y desempeño laboral docente (clúster 3). Este ultimó, fue agrupado en un solo clúster a pesar que en la teoría de Echeverría (2002) corresponde a 4 dimensiones, competencias técnicas, metodológicas, participativas y personales.

\section{Clúster 1: Motivación extrínseca y trascendental}

En el primer clúster, de acuerdo a los ítems encontramos una comunicación fluida entre el docente y el director de escuela, una comodidad en el ambiente físico de su trabajo, incentivo por su 
trabajo docente el cual contribuye a lograr los objetivos institucionales. Asimismo, el desempeño del docente benefició a sus estudiantes el cual comprendió el logro de los objetivos institucionales y hubo sinergia en relación con sus compañeros de trabajo.

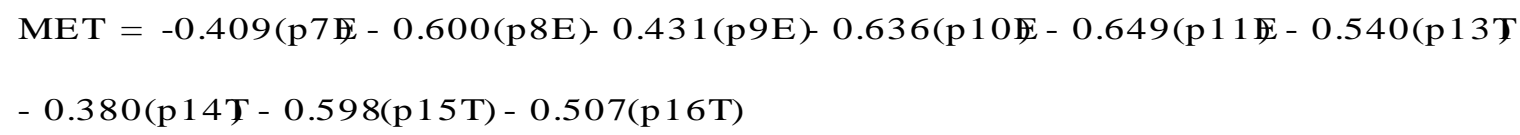

\section{Clúster 2: Motivación intrínseca}

En el segundo clúster de acuerdo a los ítems, el docente cumplió con sus expectativas personales, se sintió con energía para realizar su trabajo el cual le gusta hacer y se sintió motivado en superación personal.

$$
\mathrm{MI}=-0.449(\mathrm{p} 1 \mathrm{I}) 0.318(\mathrm{p} 2 \mathrm{H}) 0.327(\mathrm{p} 3 \mathrm{H}) 0.186(\mathrm{p} 4 \mathrm{I}) 0.296(\mathrm{p} 5 \mathrm{I})
$$

\section{Clúster 3: Desempeño docente}

En el clúster tres, de acuerdo al agrupamiento de los reactivos se observa que, el docente brinda ejemplos de los temas, explica la utilidad de los contenidos, establece estrategias para alcanzar el aprendizaje, así como usa herramientas tecnológicas de plataformas virtuales, que facilita el proceso de enseñanza y aprendizaje del estudiante. Asimismo, fomenta y elogia por los méritos alcanzados en sus estudiantes, así como realizó un trabajo unificado y colaborativo entre sus compañeros. Además, la puntualidad fue importante en la participación de las actividades institucionales.

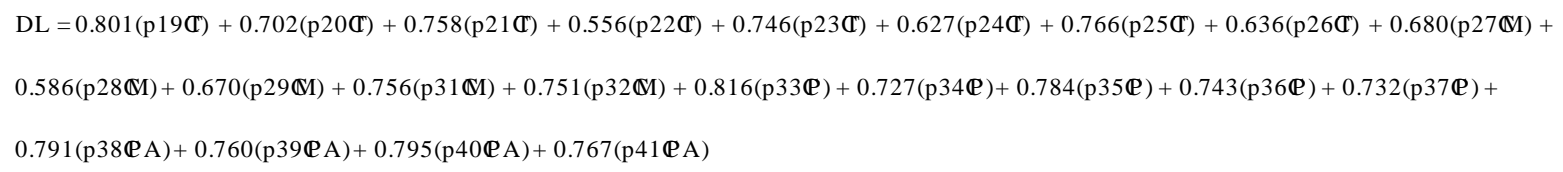




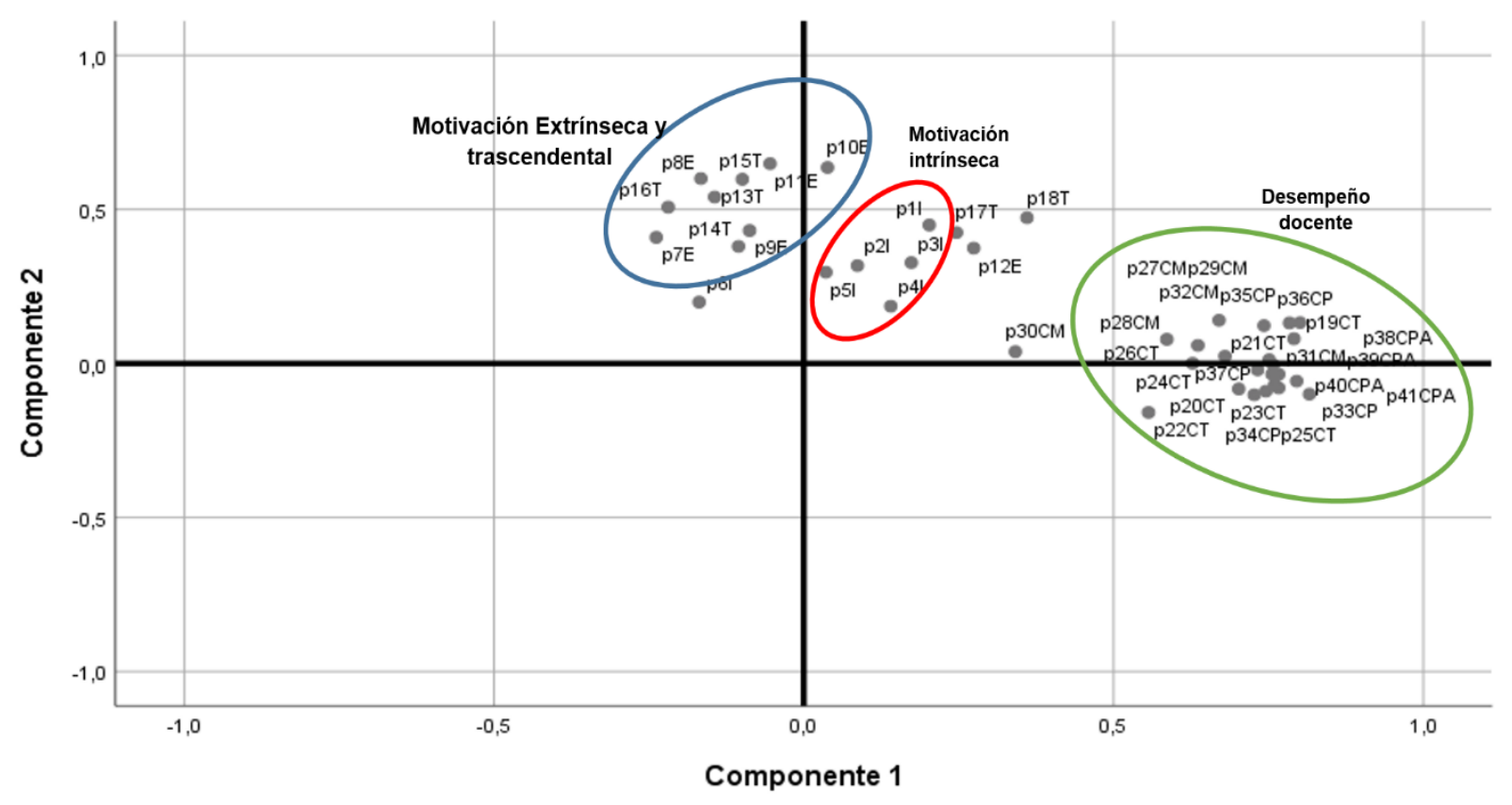

Figura 8. Componentes principales rotado con Varimax

\section{Modelo de componente factorial de la investigación}

Para el cumplimiento del objetivo del presente estudio, se procedió a realizar la extracción de ítems de los constructos motivación y desempeño docentes, con valores propios mayores que 1 y una varianza explicada del 39,61\%, se obtuvo los modelos factoriales representados en la tabla 5 con la técnica de rotación del Varimax. 
Tabla 5

Modelo de componente rotados

\begin{tabular}{|c|c|c|c|}
\hline Ítems & Factor1 & Factor2 & Comunalidad \\
\hline $\mathrm{p} 1 \mathrm{I}$ & 0.203 & -0.449 & 0.243 \\
\hline $\mathrm{p} 2 \mathrm{I}$ & 0.087 & -0.318 & 0.108 \\
\hline p3I & 0.174 & -0.327 & 0.137 \\
\hline $\mathrm{p} 4 \mathrm{I}$ & 0.141 & -0.186 & 0.054 \\
\hline p5I & 0.036 & -0.296 & 0.089 \\
\hline p6I & -0.169 & -0.199 & 0.068 \\
\hline $\mathrm{p} 7 \mathrm{E}$ & -0.238 & -0.409 & 0.224 \\
\hline $\mathrm{p} 8 \mathrm{E}$ & -0.166 & -0.600 & 0.388 \\
\hline p9E & -0.088 & -0.431 & 0.194 \\
\hline $\mathrm{p} 10 \mathrm{E}$ & 0.038 & -0.636 & 0.406 \\
\hline $\mathrm{p} 11 \mathrm{E}$ & -0.054 & -0.648 & 0.424 \\
\hline $\mathrm{p} 12 \mathrm{E}$ & 0.274 & -0.374 & 0.215 \\
\hline $\mathrm{p} 13 \mathrm{~T}$ & -0.144 & -0.540 & 0.313 \\
\hline $\mathrm{p} 14 \mathrm{~T}$ & -0.105 & -0.380 & 0.155 \\
\hline $\mathrm{p} 15 \mathrm{~T}$ & -0.099 & -0.598 & 0.367 \\
\hline $\mathrm{p} 16 \mathrm{~T}$ & -0.219 & -0.507 & 0.305 \\
\hline $\mathrm{p} 17 \mathrm{~T}$ & 0.246 & -0.425 & 0.241 \\
\hline $\mathrm{p} 18 \mathrm{~T}$ & 0.360 & -0.473 & 0.354 \\
\hline p19CT & 0.801 & -0.132 & 0.659 \\
\hline $\mathrm{p} 20 \mathrm{CT}$ & 0.702 & 0.083 & 0.500 \\
\hline $\mathrm{p} 21 \mathrm{CT}$ & 0.758 & 0.005 & 0.574 \\
\hline $\mathrm{p} 22 \mathrm{CT}$ & 0.556 & 0.159 & 0.335 \\
\hline $\mathrm{p} 23 \mathrm{CT}$ & 0.746 & 0.090 & 0.564 \\
\hline $\mathrm{p} 24 \mathrm{CT}$ & 0.627 & -0.000 & 0.394 \\
\hline $\mathrm{p} 25 \mathrm{CT}$ & 0.766 & 0.079 & 0.594 \\
\hline $\mathrm{p} 26 \mathrm{CT}$ & 0.636 & -0.059 & 0.408 \\
\hline $\mathrm{p} 27 \mathrm{CM}$ & 0.680 & -0.023 & 0.463 \\
\hline $\mathrm{p} 28 \mathrm{CM}$ & 0.586 & -0.078 & 0.349 \\
\hline $\mathrm{p} 29 \mathrm{CM}$ & 0.670 & -0.140 & 0.469 \\
\hline p30CM & 0.341 & -0.038 & 0.118 \\
\hline $\mathrm{p} 31 \mathrm{CM}$ & 0.756 & 0.035 & 0.573 \\
\hline $\mathrm{p} 32 \mathrm{CM}$ & 0.751 & -0.012 & 0.564 \\
\hline $\mathrm{p} 33 \mathrm{CP}$ & 0.816 & 0.100 & 0.676 \\
\hline $\mathrm{p} 34 \mathrm{CP}$ & 0.727 & 0.102 & 0.539 \\
\hline $\mathrm{p} 35 \mathrm{CP}$ & 0.784 & -0.132 & 0.632 \\
\hline $\mathrm{p} 36 \mathrm{CP}$ & 0.743 & -0.123 & 0.567 \\
\hline $\mathrm{p} 37 \mathrm{CP}$ & 0.732 & 0.021 & 0.537 \\
\hline p38CPA & 0.791 & -0.080 & 0.632 \\
\hline p39CPA & 0.760 & 0.069 & 0.583 \\
\hline p40CPA & 0.795 & 0.057 & 0.636 \\
\hline p41CPA & 0.767 & 0.035 & 0.589 \\
\hline Varianza & 12.368 & 3.872 & 16.240 \\
\hline$\%$ Var & 0.302 & 0.094 & 0.396 \\
\hline
\end{tabular}

En la figura 9, se observa un centroide ligado a los ítems por dimensiones, que utiliza la distancia euclidiana, donde los ítems más próximos al centroide son los más óptimos. Existen tres agrupaciones que revelan el efecto de las variables en estudio; es decir, en la figura se observa los gráficos de segmentos de ítems. 
El primer segmento, se observa un patrón ligado al desempeño docente distante al centroide, en la que el ítem con menor distancia al centroide aporta más al desempeño docente. Esto significa que su desempeño destaca en actividades complementarias con metas más altas, ellos sienten la necesidad de seguir superándose en el ámbito profesional, con el ánimo de realizar su trabajo docente.

El segundo segmento, se observa un patrón ligado a la motivación intrínseca con una diferencia menor que el desempeño con relación al centroide, en la que los ítems cercanos aportan más a la motivación intrínseca. Asimismo, un tercer segmento, se observa un patrón de agrupamiento de dos conceptos ligadas a la motivación extrínseca y trascendental en función al centroide, donde los ítems cercanos al centroide contribuyen más a los conceptos mencionados.

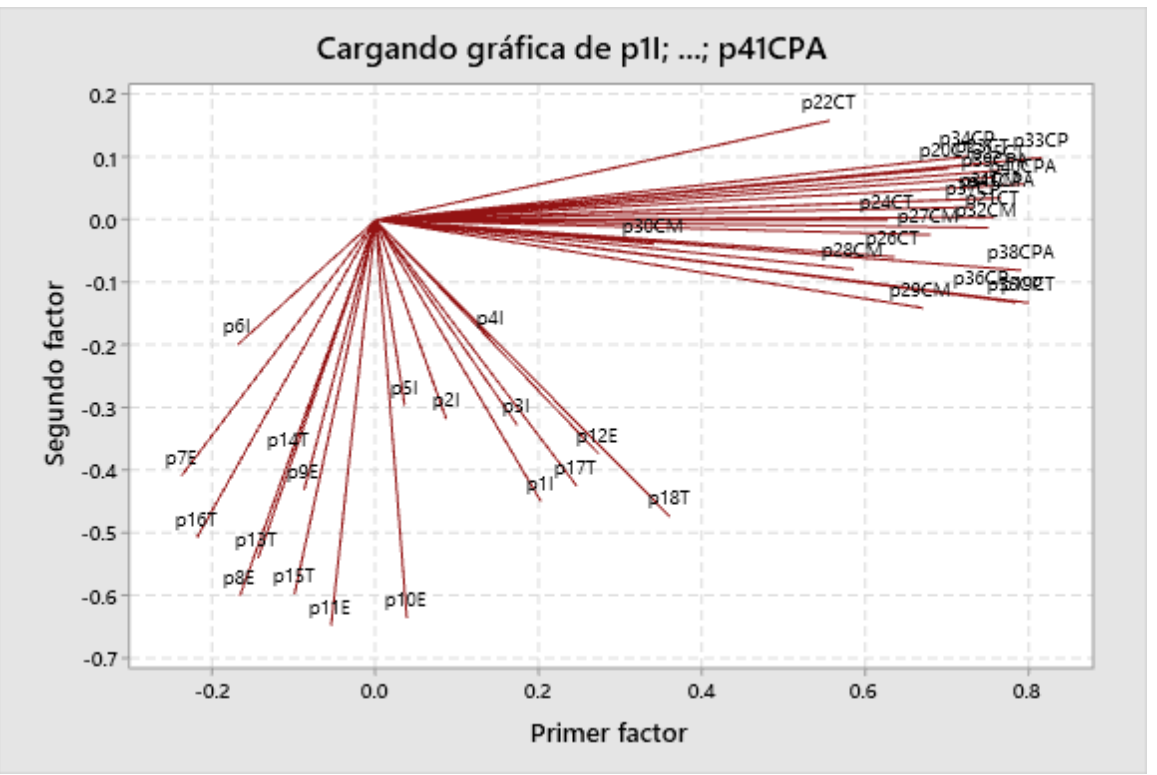

Figura 9. Gráfica de factores

\section{Discusión}

El objetivo del presente estudio fue analizar los indicadores de la motivación y desempeño docente para determinar un modelo factorial de predicción en docentes, utilizando una metodología cuantitativa predictiva que permitió realizar un análisis factorial en los constructos.

Los resultados arrojaron tres agrupamientos: motivación extrínseca y trascendental (clúster 1), motivación intrínseca (clúster 2) y desempeño docente (clúster 3). Esto permitió analizar una varianza explicada con valores propios mayores que uno, donde se obtuvieron dos modelos de la componente que predicen el comportamiento de los constructos, cuya arquitectura se muestra en la 
tabla 5 .

Los resultados evidencian que la motivación influye en el desempeño docente y bajo los modelos factoriales según las ecuaciones antes mencionadas permiten predecir de la motivación y de desempeño. En esta línea, se puede mencionar que los resultados coinciden con los propuestos por Villagra, Mellado, \& Cubo (2018), donde menciona que la motivación trasciende en la medida que favorece un mejor desempeño de los docentes y viceversa. Asimismo, Gutiérrez (2014) concluye que la motivación y el desempeño tiene alta relación entre los constructos. Se infiere que la motivación tiene una gran importancia para predecir un eficiente desempeño docente, basado en competencias técnicas, metodológicas, participativas y personales.

Indudablemente el éxito de cualquier institución educativa es lograr tener una comunidad académica con buen rendimiento educativo en sus competencias, en cambio sí existe rencillas entre docentes por diversas circunstancias, será difícil brindar calidad de enseñanza educativa (FrancoLópez, Vélez, \& López-Arellano, 2018). Para el caso de la Institución Educativa Emblemática Isabel la Católica, el éxito de la institución está garantizado en la varianza explicada de las dos componentes de un 39.609\% con dos valores propios mayores que uno y una rotación Varimax que permitió mejorar el rendimiento de los reactivos.

Es preciso indicar que las experiencias positivas del docente producen satisfacción, conllevan al desarrollo y realización profesional, que tiene un efecto significativo en la enseñanza y el aprendizaje del estudiante (González \& Subaldo, 2015). En ese sentido, la administración de la institución educativa está llamada a establecer estrategias de motivación extrínseca y trascendental en el personal docente, con el objetivo de predecir un mejor desempeño docente. Sin embargo, el desempeño del profesorado se verá afectado por su motivación intrínseca, siendo la institución la responsable de identificar esos motivadores intrapersonales para aprovechar y beneficiar mejora a sus docentes.

Está comprobado que la vocación es el factor intrínseco que tiene mayor impacto en la motivación del docente y con un efecto menor el reconocimiento económico. El conjunto de conocimientos que el docente pone al servicio del estudiante llega hacer un motivante intrínseco muy relevante (Franco-López et al., 2018). Asimismo, las experiencias satisfactorias del docente repercuten significativamente en la vida personal y profesional. Sus experiencias positivas conllevan al crecimiento y a mejorar la calidad del proceso de la enseñanza y aprendizaje en los estudiantes, además, al compromiso con su vocación, a la institución educativo, y la felicidad y bienestar del 
mismo (González \& Subaldo, 2015)

\section{Conclusión}

Se concluye que al analizar los indicadores de la motivación y desempeño permitieron determinar, no solo un modelo factorial, sino dos modelos que ayudan a predecir los resultados en cuanto a estos dos constructos, logrando en los investigadores aseverar que las dimensiones de la motivación no solo influyen en el desempeño, sino que permite predecir buenos resultados en el desempeño docente para el caso de la IEE. Isabel la Católica. Sin lugar a duda, la motivación está comprobada que es esencial para el éxito en el aprendizaje y el proceso de enseñanza. Lo que determina que la motivación del docente está profundamente relacionada con lo que sucede en el salón de clase y con el entorno social del estudiante (Rodríguez-Pérez, 2012).

Reflexionar que el docente es el mentor, líder y facilitador de la formación y preparación de sus estudiantes de manera reflexiva, crítica e innovadora, que contribuye a formar ciudadanos participativos, transformadores de una sociedad democrática, justa y tolerante, basada en el respeto y los derechos que es posible alcanzar con un buen desempeño en todas sus dimensiones que contribuirán al perfeccionamiento de la anhelada excelencia de la educación. Precisar que estos modelos de predicción son válidos para el caso de la Institución Educativa Emblemática Isabel la Católica. Sin embargo, se recomienda que puedan ser probados en otras instituciones, pero es necesario considerar otras variables en cuestión para ser más asertivos en la predicción de las dimensiones de la motivación y desempeño en los docentes.

Finalmente, es propicio mencionar que por ser un estudio que partió de la asignatura Herramientas para la Investigación con estudiantes del 5to ciclo, no se contó con suficiente presupuesto para ampliar el estudio a otras instituciones del mismo nivel educativo, por lo que se recomienda que para futuras investigaciones esto logre ampliarse y ver si el modelo predictivo pueda ser adaptado o logre estandarizarse.

\section{Referencias}

Aneas, A. (2003). Competencias profesionales. Análisis conceptual y aplicación profesional. Seminari Permanent d'Orientació Professional, 1-16. Retrieved from http://www.edu.xunta.gal/centros/cfrcoruna/system/files/Asumpta+Aneas.pdf

Blumen, S. (2008). Motivación, sobredotación y talento: un desafío para el éxito. Revista de Psicología, XXVI(1), 147-184. Retrieved from 
http://pepsic.bvsalud.org/pdf/rp/v26n1/v26n1a08.pdf

Bruno, A. M. (2017). Motivación laboral y desempeño docente de las maestras de educación inicial de la Red 03-UGEL No 07 - 2016 (Universidad César Vallejo). Retrieved from http://repositorio.ucv.edu.pe/handle/20.500.12692/8258

Catena, A., Ramos, M. M., \& Trujillo, H. M. (2003). Análisis multivariado. Un manual para investigadores. Madrid: Biblioteca Nueva.

Cedeno, A. (2004, July 5). Opinion - Responsabilidad social de la empresa. NoticiasFinancieras, pp. 1-3. Retrieved from https://search.proquest.com/docview/468338899?accountid=12268\%0A

Centro Europeo para el Desarrollo de la Formación Profesional. (2010). Modernización de la formación profesional. Cuarto informe de investigación sobre la formación profesional en Europa:resumen. Luxemburgo: Oficina de Publicaciones de la Unión Europea.

César, P. (2004). Técnicas de Análisis Multivariante de Datos aplicaciones con SPSS (1st ed.). Madrid: Pearson Educación.

Chiavenato, I. (2014). Comportamento organizacional: a dinamica do sucesso das organizaçoes (3rd ed.). Brasil: Manole.

Echeverría, A. (2002). Gestión de la Competencia de Acción Profesional. Revista de Investigación Educativa, 20(1), 7-46. Retrieved from https://revistas.um.es/rie/article/view/97411

Franco-López, J. A., Vélez, F. M., \& López-Arellano, H. (2018). La motivación docente y su repercusión en la calidad educativa: estudio de caso. Revista de Pedagogía, 39(105), 151172. Retrieved from https://www.researchgate.net/publication/334466394

Franco, J. A., López, H., \& Vélez, M. (2015). La influencia de la motivación docente en el proceso de aprendizaje. El Análisis Organizacional En México y América Latina. Retos y Perspectivas a 20 Años de Estudios, 2201-2240. Retrieved from https://www.researchgate.net/publication/283721987 
Gálvez, E., \& Milla, R. (2018). Evaluación del desempeño docente: Preparación para el aprendizaje de los estudiantes en el Marco de Buen Desempeño Docente. Propósitos y Representaciones, 6(2), 407-452. https://doi.org/10.20511/pyr2018.v6n2.236

Gómez, L. F., \& Valdés, M. G. (2019). La evaluación del desempeño docente en la educación superior. Propósitos y Representaciones, 7(2), 479-497. https://doi.org/10.20511/pyr2019.v7n2.255

González, J., \& Subaldo, L. (2015). Opiniones sobre el desempeño docente y sus repercusiones en la satisfacción profesional y personal de los profesores. Educación, 24(47), 90-114. Retrieved from http://repositorio.minedu.gob.pe/handle/123456789/4051

Guerrero, D. A., La Rosa, G., \& Díaz, N. (2015). Analysis of Research Literature of Professional Competency Models with a Cognitive-motivational Approach. Procedia - Social and Behavioral Sciences, 171, 1400-1409. https://doi.org/10.1016/j.sbspro.2015.01.260

Gutiérrez, M. (2014). La motivación y el desempeño laboral de los docentes de la Institución Educativa Domingo Mandamiento Sipan, Hualmay - 2014 (Universidad César Vallejo). Retrieved from https://hdl.handle.net/20.500.12692/4831

Jiménez, A., \& Bueno, J. L. (2003). Qué motiva a sus colaboradores? : descúbralo y mejore el rendimiento : no hay recetas. Madrid: Fundación Confemetal.

Lewis-Beck, M., Bryman, A., \& Futing, T. (2004). The SAGE Encyclopedia of Social Science Research Methods. In The SAGE Encyclopedia of Social Science Research Methods. https://doi.org/10.4135/9781412950589

López-Arellano, H., Vélez-Salazar, M., \& Franco-López, J. A. (2017). Percepciones acerca de la motivación docente en personal directivo de instituciones de educación secundaria en la zona metropolitana de Medellín, 2015. Revista Electrónica Educare, 21(2), 1-23. https://doi.org/10.15359/ree.21-2.5

Martínez-Chairez, G. I., Guevara-Araiza, A., \& Valles-Ornelas, M. M. (2016). El desempeño docente y la calidad educativa. Ra Ximhai, 12(6), 123-134. https://doi.org/10.35197/rx.12.01.e3.2016.06.gm 
Martínez, F. M., \& Carmona, G. (2009). Aproximación al concepto de "competencias emprendedoras": valor social e implicaciones educativas. Revista Iberoamericana Sobre Calidad, Eficacia y Cambio En Educación, 7(3), 82-98. Retrieved from http://www.rinace.net/reice/numeros/arts/vol7num3/art6.pdf

Oficina Internacional del Trabajo. (2005). Recomendación 195. Recomendación sobre el Desarrollo de los Recursos Humanos: Educación, Formación y Aprendizaje Permanente (1st ed.). Ginebra: Oficina Internacional del Trabajo.

Pacheco, A. G. (2016). El acompañamiento pedagógico de los directores y el desempeño laboral de los docentes de las instituciones educativas de educación primaria del distrito de José Luis Bustamante y Rivero, Arequipa 2016 (Universidad Nacional de San Agustín). Retrieved from http://repositorio.unsa.edu.pe/handle/UNSA/2467

Rodríguez-Pérez, N. (2012). La motivación del profesor: un factor fundamental para la eficacia del proceso de enseñanza-aprendizaje de idiomas. Encuentro: Revista de Investigación e Innovación En La Clase de Idiomas, (21), 126-142. Retrieved from https://ebuah.uah.es/dspace/handle/10017/14861

Rojas-Segovia, M. M., \& Romero-Varela, D. Y. (2019). Revisión de la influencia de la motivación docente en el empleo de las pizarras digitales interactivas. Propósitos y Representaciones, 7(2), 516-525. https://doi.org/10.20511/pyr2019.v7n2.228

Saini, M., Kumar, A., \& Kaur, G. (2020). Research Perception, Motivation and Attitude among Undergraduate Students: A Factor Analysis Approach. Procedia Computer Science, 167, 185-192. https://doi.org/10.1016/j.procs.2020.03.210

Sánchez-Claros, J. P. (2014). Influencia de la cultura académica de origen en el desempeño docente de profesores universitarios noveles. Historia y Comunicación Social, 19(Esp. Febrero), 523-533. https://doi.org/10.5209/rev_HICS.2014.v19.45046

Sánchez, S. L. (2019). Motivación docente y gestión educativa, en instituciones educativas públicas, UGEL 02, Los Olivos 2018 (Universidad César Vallejo). Retrieved from http://repositorio.ucv.edu.pe/handle/20.500.12692/27072 
Sanizaca, B. (2018). Reflexiones acerca de la motivación. MAPA Revista de Ciencias Sociales y Humanística, 2(8), 226-233. Retrieved from http://www.revistamapa.org/index.php/es/article/view/61

Schnitker, S. A., Gilbertson, M. K., Houltberg, B., Hardy, S. A., \& Fernandez, N. (2020). Transcendent motivations and virtue development in adolescent marathon runners. Journal of Personality, 88(2), 237-248. https://doi.org/10.1111/jopy.12481

Silva, M. (2010). Assessing Leadership Skills Leaders in Performance Intermediate/Organisational Effectiveness Case Study of a Multinational Pharmaceutical Branch. University of Coimbra Press.

Sucuoglu, E. (2017). Analysis of motivational strategies used by English language teachers teaching at secondary schools. Procedia Computer Science, 120, 189-195. https://doi.org/10.1016/j.procs.2017.11.228

Ugarte, C., \& Naval, C. (2010). Developing Professional Competences in Higher Education. A Case Study. Revista Electronica de Investigacion Educativa, 12(Número Especial), 1-14. Retrieved from https://redie.uabc.mx/redie/article/view/251

Velasco, E. M., Bautista, H., Sánchez, F., \& Cruz, L. (2012). La motivación como factor de influencia en el desempeño laboral del área docente del instituto tecnológico superior de Tantoyca (Instituto Tecnológico Superior de Tantoyuca). Retrieved from http://www.eumed.net/libros-gratis/2011c/992/index.htm

Villagra, C., Mellado, M. E., \& Cubo, S. (2018). Relación entre variables mediadoras del desempeño docente y resultados educativos : una aproximación al liderazgo escolar. Opción: Revista de Ciencias Humanas y Sociales, 34(87), 213-240. Retrieved from https://produccioncientificaluz.org/index.php/opcion/article/view/23878 